\title{
Experimental Implementation of a Photonic Neural Network with a 1550nm-VCSEL subject to Optical Injection and Delayed Optical Feedback
}

\author{
Julián Bueno, Joshua Robertson, Matěj Hejda, and Antonio Hurtado \\ Institute of Photonics, Dept. of Physics, University of Strathclyde, Technology and Innovation Centre, Glasgow, UK
}

\begin{abstract}
We investigate experimentally the performance of an ultrafast photonic reservoir computer based on an off-the-shelf 1550nm-VCSEL subject to optical injection and delayed optical feedback, creating an artificial network of hundreds of GHz-speed neurons. We also demonstrate successful operation in different complex processing tasks.
\end{abstract}

Keywords—VCSEL, artificial intelligence, reservoir computing, optical delayed feedback, signal processing

\section{INTRODUCTION}

Society's computing requirements have exponentially increased over the last decades, both in terms of quantity of data and complexity of processing tasks. The improvement of classical computing platforms based on electrical transistors is slowing down due to fundamental physical limitations. This challenge has increased the interest in non-conventional computing techniques such as artificial neural networks (ANN), which over the last decade have proven to be extremely successful to solve problems for which classical computing architectures struggle (e.g. pattern and image recognition tasks). Therefore, these novel computing techniques will help alleviate the current information processing load by efficiently and successfully solving highly complex tasks. Importantly, ANNs rely on contrasting hardware implementations compared to traditional transistor-based digital computer architectures and which have proven to be highly hardware friendly for implementations based in photonic technologies. Thus, in recent years, different photonic ANN hardware implementations have been demonstrated using a variety of devices and approaches $[1,2]$

Among photonic ANN realisations, reservoir computing techniques have undergone considerable attention [3]. These approaches rearrange the hidden layers of the ANN into a reservoir, substantially simplifying the learning stages whilst also creating intrinsic memory inside the ANN (an extremely helpful feature for a wide variety of processing tasks). Moreover, reservoir computers (RC) can be implemented by using a single nonlinear element and a delay line [4]. These concepts have been extremely useful to develop multiple photonic and optical neural networks experimentally implemented in hardware based on different devices including semiconductor lasers [5-7]. Yet, to date these have been mostly based on in-plane laser sources, and have not focused on verticalcavity surface emitting lasers (VCSELs). Only very recently the first reports have emerged predicting theoretically enhanced performance in VCSEL-based reservoir computing ANNs by exploiting the unique light polarisation properties of these devices [8]. In this work, we experimentally explore the properties and capabilities of a RC ANN system based on a VCSEL emitting at the important telecom wavelength of $1550 \mathrm{~nm}$ and its dependence on different experimental parameters.

\section{EXPERIMENTAL SETUP AND METHODS}

The experimental setup used in this work to build the VCSEL-based RC ANN is depicted in Fig. 1. It consists of off-the-shelf fibre-optic components, including a VCSEL emitting at $1546 \mathrm{~nm}$ and with a lasing threshold current of $1.82 \mathrm{~mA}$ (at 293K). The VCSEL's spectrum showed two orthogonally polarised modes, the main lasing mode (parallel-polarised mode) and a subsidiary attenuated mode (orthogonally-polarised mode). These were separated by $0.11 \mathrm{~nm}$ and corresponded to the two orthogonal polarizations of the fundamental transverse mode of the device. The device is subject to delayed optical feedback using an optical circulator. The delay feedback time was measured to be $65.6 \mathrm{~ns}$; hence by injecting one sample every delay time this system currently processes information at $15 \mathrm{MHz}$. A Variable Optical Attenuator (VOA) and a Polarization Controller (PC) are used to control the intensity and polarization (parallel or orthogonally-polarised) of the optical feedback, respecively. The optical feedback reduced the threshold current by $8.1 \%$ for parallel-polarised feedback and $2.7 \%$ for rotated orthogonally-polarised feedback. Optical couplers are used to inject information optically into the VCSEL and to extract part of the feedback light for detection. A tunable laser provides $\mathrm{CW}$ light and information is encoded by modulating its intensity with a Mach-Zehnder modulator. A second VOA and PC are used to control the intensity and polarization (parallel or orthogonal) of the injected light into the VCSEL. The percentage of output light sent for detection is both optically and electrically amplified to improve the Signal to Noise Ratio (SNR) before it is recorded with an $8 \mathrm{GHz}$ bandwidth and $20 \mathrm{Gsa} / \mathrm{s}$ oscilloscope.

The injected signal consists of a temporal mask that is continuously iterated every delay time ( $65.6 \mathrm{~ns})$. In each iteration the mask is multiplied by the value that is to be injected into the ANN. The mask consisted of 328 values randomly selected from the subset $\{-$ $1,-0.6,-0.2,+0.2,+0.6,+1\}$, modulated at $5 \mathrm{GSa} / \mathrm{s}$ using a $5 \mathrm{GHz}$ Arbitrary Waveform Generator. This creates an ANN with 328 virtual nodes ( $200 \mathrm{ps}$ long each) inside the total temporal length of the delay feedback loop. Output weights are computed offline via a supervised learning algorithm, considering an output layer with one node. 


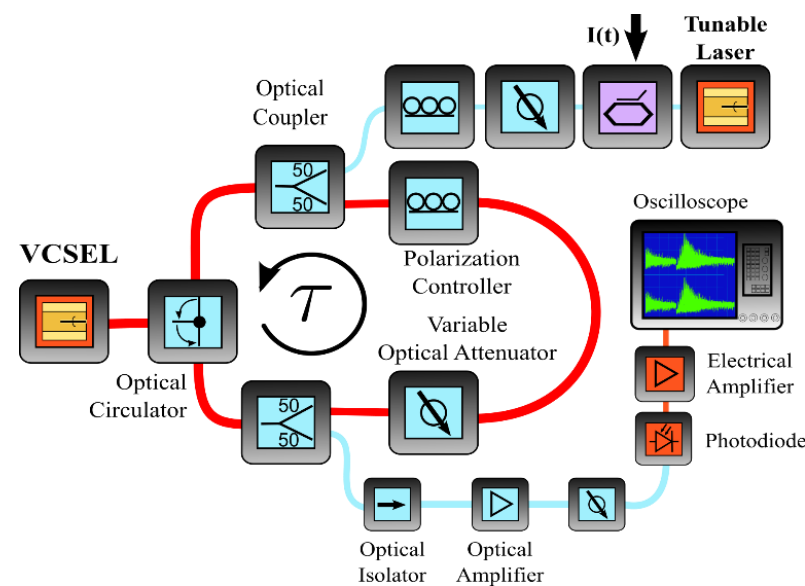

Figure 1. Diagram of the experimental setup used to build a $R C$ system with a $1550 \mathrm{~nm}$-VCSEL.

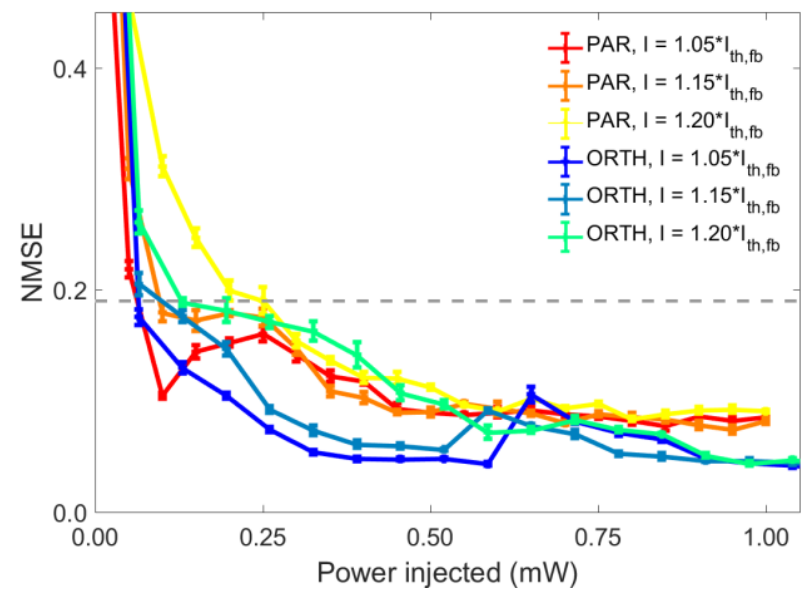

Figure 2. Measured performance of the VCSEL RC system for the Mackey-Glass prediction task.

\section{RESULTS AND CONCLUSSIONS}

To evaluate the performance of the 1550nm-VCSEL RC ANN system of this work we initially use the Mackey-Glass Prediction Task using the NMSE (Normalized Mean Square Error). In this task, the ANN has to predict the next value in a chaotic time-series based on its previous history. Ten thousand input values and 5-fold cross-validation were used to ensure adequate learning conditions. Fig. 2 shows the performance of the 1550nm-VCSEL RC system. The NMSE was experimentally measured for different injected powers under diverse applied bias currents and injected light polarisation conditions. The injected power plotted in Fig. 2 was measured right after the optical coupler used in the optical injection line. The polarization of the optical injection and delayed feedback light was set parallel (PAR) or orthogonal (ORTH) with respect to that of the main lasing mode of the 1550nm-VCSEL. The gray dashed line in fig. 2 (at NMSE $=0.19$ ) indicates the performance of a linear reservoir on this task. Fig. 2 shows that for very low injected powers the error is large regardless of the operating conditions. As power increases, the error reduces quickly reaching low values of approx. NMSE $=0.05$ for ORTH and NMSE $=0.075$ for PAR. This reduction in the achieved error is due to the VCSEL being stably locked to the external-injection which increases the response consistency, whilst the observed improved performance for the orthogonally-polarised configuration is in agreement with recently reported theoretical predictions [8]. Fig. 2 also shows that for lower currents closer to threshold, the VCSEL RC system exhibits better performance when lower optical injection powers are configured. This is due to the lower power requirements needed to achieve injection locking as bias current reduces. These results experimentally demonstrate that VCSEL-based RC neural networks exhibit a broad parameter region with successful performance on general prediction tasks. Moreover, the unique polarisation properties of VCSELs enable an extra operation degree of freedom permitting the use of different polarization schemes for enhanced performance.

\section{ACKNOWLEDGMENT}

The authors thank support from the US Office of Naval Research Global (Grant ONRG-NICOP-N62909-18-1-2027), the European Commission (Grant 828841-ChipAI-H2020-FETOPEN-2018-2020) and the EPSRC Doctoral Training Partnership (EP/N509760).

\section{REFERENCES}

[1] J. Feldmann, N. Youngblood, C. D. Wright, H. Bhaskaran, and W. H. P. Pernice, "All-optical spiking neurosynaptic networks with self-learning capabilities," Nature, vol. 569, issue 7755, 2019, pp. 208-214.

[2] X. Lin, Y. Rivenson, N. T. Yardimci, M. Veli, Y. Luo, M. Jarrahi, and A. Ozcan, "All-optical machine learning using diffractive deep neural networks," Science, vol. 8084, July 2018.

[3] H. Jaeger, H. Haas, "Harnessing Nonlinearity: Predicting Chaotic Systems and Saving Energy in Wireless Communication," Science, vol. 304, issue 5667, 2004, pp. 78-80.

[4] L. Appeltant, M. C. Soriano, G. Van Der Sande, J. Danckaert, S. Massar, J. Dambe, B. Schrauwen, C. R. Mirasso, and I. Fischer, "Information processing using a single dynamical node as complex system," Nature Communications, vol. 2, issu 1, 2011.

[5] D. Brunner, M. C. Soriano, C. R. Mirasso, and I. Fischer, "Parallel photonic information processing at gigabyte per second data rates using transient states," Nature Communications, vol. 4, 2013.

[6] J. Bueno, S. Maktoobi, L. Froehly, I. Fischer, M. Jacquot, L. Larger, and D. Brunner, "Reinforcement learning in a large-scale photonic recurrent neural network," Optica, vol. 5, issue 6, 2018, pp. 756-760.

[7] P. Antonik, N. Marsal, D. Brunner, and D. Rontani, "Human action recognition with a large-scale brain-inspired photonic computer," Nature Machine Intelligence, vol. 1, issue 11, 2019, pp. 530-537.

[8] J. Vatin, D. Rontani, and M. Sciamanna, "Enhanced performance of a reservoir computer using polarization dynamics in VCSELs," Optics Letters, vol. 43, issue 18, 2018, pp. 4497-4500. 